\title{
Radiology in the lead: towards radiological profiling for precision medicine in glioblastoma patients? Editorial comment on Glioblastoma patients with a moderate vascular profile benefit the most from MGMT methylation
}

\author{
Bart Roelf Jan van Dijken ${ }^{1}$ (D) A. van der Hoorn ${ }^{1}$ \\ Received: 20 October 2020 / Revised: 2 November 2020 / Accepted: 2 December 2020 / Published online: 11 January 2021 \\ (C) European Society of Radiology 2021
}

Primary brain tumors, most commonly of glial origin, are responsible for a high disease burden and loss of healthy life years. Glioblastoma (GBM), being the most malignant and most frequently occurring subtype, has a median survival of only 14.6 months [1]. This devastating number has changed little over the past two decades. The last significant step forward for GBM patients came with the incorporation of temozolomide (TMZ) chemotherapy in standard treatment protocol, increasing the median overall survival with 2.5 months when compared to radiotherapy alone [1]. A need for novel treatment strategies for GBM patients clearly remains.

The paradigm shift of "one fits all" to "all fit one" associated with precision medicine has therefore in recent years also emerged in neuro-oncological treatment schemes. After all, it is rather peculiar to implement homogeneous treatment protocols for such a heterogeneous population. Molecular markers and genetic profiles now play a pivotal role in the management of GBM [2]. For instance, as suggested by an upcoming guideline update, certain molecular parameters such as TERT promoter mutation, EGFR gene amplification, or chromosome 7 gain define GBM even if histologically the tumor is suggestive of a lower grade tumor [3]. Thus, current and future guidelines emphasize the heterogeneity and interpatient variability of GBM.

Currently, one of the most widely accepted molecular markers for prognostication and treatment decision-making

This comment refers to the article available at https://doi.org/10.1007/ s00330-020-07297-4

Bart Roelf Jan van Dijken

b.r.j.van.dijken@umcg.nl

1 Department of Radiology, Medical Imaging Center (MIC), University Medical Center Groningen, Hanzeplein 1, 9700, RB Groningen, the Netherlands in GBM is O6-methylguanine-DNA methyltransferase (MGMT) methylation status. MGMT-methylated tumors are more susceptible to alkylating chemotherapeutic agents such as TMZ, leading to an improved prognosis. As a result, current guidelines suggest the addition of TMZ complementing radiotherapy for elderly patients (> 70 years) with MGMTmethylated GBM only [4].

Published in this issue, a study by Fuster-Garcia and colleagues, however, has found that the prognostic impact of MGMT methylation might be influenced by the tumor's vascularity [5]. In their multicenter retrospective study among 96 GBM patients, the authors have demonstrated that there is a beneficial effect of MGMT methylation in tumors with moderately vascular status only. Only a non-significant trend was found for patients with highly vascularized MGMTmethylated GBM. These results are indicative of certain subpopulations of MGMT-methylated GBM that are more susceptible to treatment than others. The authors correctly claim that not considering such information could potentially induce bias in future clinical studies.

For the abovementioned study, the authors used an open online segmentation service (ONCOhabitats) to identify different regions within the tumor based on conventional anatomical sequences as well as perfusion MRI. These regions (habitats) were identified, by fusing anatomical information of the tumor (the contrast-enhancing tumor bulk and peritumoral edema) with the perfusion data, leading to moderately vascularized and highly vascularized tumor habitats.

The habitat approach is drawn from the idea that GBM is not a homogenous entity but a rather heterogeneous tumor. Habitat approaches based on advanced MRI sequences such as perfusion or diffusion have been successfully applied by other studies $[6,7]$. Certain habitats experience more aggressive features such as high perfusion and low diffusion, suggestive of a highly vascularized and cellular tumor component. Presence of such 
aggressive tumor habitats have been shown to have a negative impact on survival [8] and treatment response [9]. In addition, certain habitats have been suggested to play a role in resistance to chemotherapy and radiotherapy and thus might in part be responsible for treatment failure [10]. Identification of such tumor habitats and thus radiological "profiling" of the individual tumor could potentially guide subsequent treatment in addition to molecular biomarkers.

To conclude, certain biomarkers such as MGMT methylation status have been given a more prominent role in the management of GBM patients over recent years. However, as Fuster-Garcia and colleagues have demonstrated, in addition to this interpatient heterogeneity, intratumor heterogeneity also plays an important role in GBM. When moving towards a precision medicine approach, such information should indeed be considered, both in future clinical studies but also in the multidisciplinary management of GBM patients. Perhaps the radiologist should be in the lead to allow personalized treatment?

Funding The authors state that this work has not received any funding.

\section{Compliance with ethical standards}

Guarantor The scientific guarantor of this publication is BRJ van Dijken, MD.

Conflict of interest The authors of this manuscript declare no relationships with any companies, whose products or services may be related to the subject matter of the article.

Statistics and biometry No complex statistical methods were necessary for this paper.

Informed consent Written informed consent was not required for this study because it is an editorial comment.

Ethical approval Institutional Review Board approval was not required because it is an editorial comment.

\section{References}

1. Stupp R, Mason WP, van den Bent MJ et al (2005) Radiotherapy plus concomitant and adjuvant temozolomide for glioblastoma. $\mathrm{N}$ Engl J Med 352:987-996

2. Louis DN, Perry A, Reifenberger G et al (2016) The 2016 World Health Organization classification of tumors of the central nervous system: a summary. Acta Neuropathol 131:803-820

3. Louis DN, Wesseling P, Aldape K et al (2020) cIMPACT-NOW update 6: new entity and diagnostic principle recommendations of the cIMPACT-Utrecht meeting on future CNS tumor classification and grading. Brain Pathol 30:844-856

4. Perry JR, Laperriere N, O'Callaghan CJ et al (2017) Short-course radiation plus temozolomide in elderly patients with glioblastoma. N Engl J Med 376:1027-1037

5. Fuster-Garcia E, Lorente Estellés D, del Mar Álvarez-Torres M et al (2020) MGMT methylation may benefit overall survival in patients with moderately vascularized glioblastomas. Eur Radiol. https:// doi.org/10.1007/s00330-020-07297-4

6. Juan-Albarracín J, Fuster-Garcia E, Pérez-Girbés A et al (2018) Glioblastoma: vascular habitats detected at preoperative dynamic susceptibility-weighted contrast-enhanced perfusion MR imaging predict survival. Radiology. 287:944-954

7. Li C, Wang S, Yan JL et al (2019) Intratumoral heterogeneity of glioblastoma infiltration revealed by joint histogram analysis of diffusion tensor imaging. Neurosurgery. 85:524-534

8. Boonzaier NR, Larkin TJ, Matys T, van der Hoorn A, Yan JL, Price SJ (2017) Multiparametric MR imaging of diffusion and perfusion in contrast-enhancing and nonenhancing components in patients with glioblastoma. Radiology. 284:180-190

9. van der Hoorn A, Yan JL, Larkin TJ, Boonzaier NR, Matys T, Price SJ (2016) Posttreatment apparent diffusion coefficient changes in the periresectional area in patients with glioblastoma. World Neurosurg 92:159-165

10. Li C, Yan JL, Torheim T et al (2019) Low perfusion compartments in glioblastoma quantified by advanced magnetic resonance imaging and correlated with patient survival. Radiother Oncol 134:1724

Publisher's note Springer Nature remains neutral with regard to jurisdictional claims in published maps and institutional affiliations. 